\title{
Chapter 14 \\ Calculation of MRI RF-Induced Voltages for Implanted Medical Devices Using Computational Human Models
}

\author{
James E. Brown, Rui Qiang, Paul J. Stadnik, Larry J. Stotts, \\ and Jeffrey A. Von Arx
}

\subsection{Introduction}

Magnetic resonance imaging (MRI), notwithstanding its status as the preferred imaging modality for soft tissue imaging and non-ionizing radiation, is generally contraindicated for patients with active implantable medical devices (AIMDs). However, it has been estimated that within 12 months of device implantation, $17 \%$ of pacemaker patients will need an MRI [1]. In order to assess the safety of devices, AIMD manufacturers work together with MR manufacturers, regulatory scientists, and academia to develop an international technical specification [2] which identifies the potential hazards for these patients in an MR environment. This test specification covers AIMDs such as deep brain stimulators, pacemakers, cochlear implants, etc.

These hazards are separated by the specific electromagnetic field component which causes that hazard. Then, the MR conditional safety of a device can be assessed in a laboratory by generating the worst case of each field component, each of which is unlikely to be observed in a clinical setting. Computational human models (CHMs) are used for three main hazards: radiofrequency (RF)-induced heating, RF-induced malfunction, and RF-induced rectification. RF-induced energy incident on the device can be rectified by non-linear electronics, which then can represent a safety issue if unintended tissue stimulation were to occur. RF-induced malfunction is a related hazard but represents the ability of the device to operate within its acceptable tolerances and free from damage during the MRI. RF-induced heating usually refers to heating of the tissue surrounding the lead tip, a phenomenon which has been shown to be linked with resonant behavior.

There are numerous advantages of using CHMs in concert with in vitro testing for this process, rather than more extensive clinical trials [4]. As the evaluation of

J. E. Brown $(\bowtie) \cdot$ R. Qiang · P. J. Stadnik $\cdot$ L. J. Stotts $\cdot$ J. A. Von Arx

Micro Systems Engineering, Inc., Lake Oswego, OR, USA

e-mail: james.brown@biotronik.com 
MR conditional safety of AIMDs contains the interaction of three subsystems, the benefits of modeling techniques are here described in relation to these three subsystems: the patient, the MR system, and the AIMD.

Patient variability can be assessed using the Virtual Family [5], which includes 15 high-resolution CHPs. These models consist of males and females of all ages in various body weights and heights and are optimized for electromagnetic simulations. Numerical modeling provides a rapid and low cost way to extract quantities such as deposited power or temperature rise in vivo.

When a human body is present in the MRI bore, the uniform EM fields, especially the electric field, will be greatly disturbed. These accumulated field distortions produce multiple local specific absorption rate (SAR)/thermal hotspots inside the human bodies, while these local hotspots further contribute to the RF-induced voltages for AIMDs. However, since each human body is unique, the body/coil interaction of each CHM will be different. The simulated electric field map in Fig. 14.1 from a CHM inside the MRI bore illustrates this effect. As seen in the figure, the hazard area positions and strengths in each human body could be significantly different. To account for this effect, a library of multiple CHMs, which spans the population in terms of BMI, shall be selected in a safety assessment. Moreover, any tissue parameter variation of the human models can be easily addressed by the computational model.

In a clinical study, only a limited number of measurements can be performed and the size of any collected statistical extreme cases would be very limited. However, in numerical modeling, millions of simulations of any combination of patients and leads, device orientations, etc. can be easily achieved. A device-based risk analysis can then be completed utilizing such a large database.

There are various MRI coil manufacturers in the market and each manufacturer builds its own MR system, i.e., each of their coils has different geometries. To calculate the statistical extreme cases, multiple body positions inside the bore have to be investigated. This variability can be assessed through modeling in a manner beyond what is practical in a clinical setting.

In a $3 \mathrm{~T}$ system, the RF field homogeneity is usually much worse than a $1.5 \mathrm{~T}$ system. To improve the field homogeneity and picture signal-to-noise ratio (SNR), a technique called shimming has drawn extensive attention in the industry and has been implemented in commercial coils. Since the induced field distribution will be altered significantly after shimming is used, this technique has to be incorporated into the safety assessment. However, each manufacturer has its own shimming technique which is invisible to the public. Computational modeling has been shown as a very convenient way to rapidly evaluate any kind of shimming technique [7].

Through the use of CHMs, the impact of variability in device orientation, including lead pathway, can be thoroughly assessed. The use of CHMs in this process enables many more data points to be evaluated than would be practical in a clinical setting. For instance, it would be impossible (and likely unethical) to vary the lead pathway and study the resulting variability in heating near the lead tip in a single patient. Expanding a clinical study to account for all potential device variabilities would be undesirable. 
Fig. 14.1 Electric field distribution for obese male model at $64 \mathrm{MHz}$

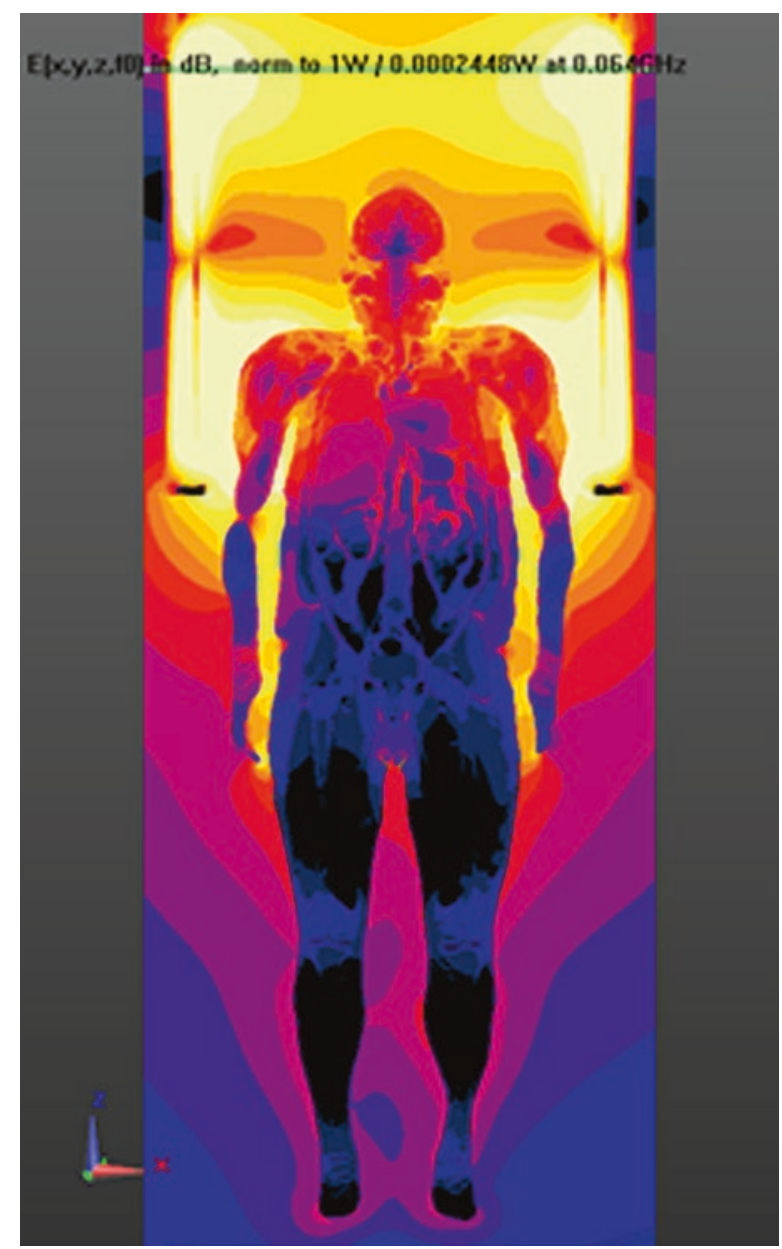

In addition, comparative statements may easily be made between AIMDs that have been modeled within the same set of CHMs. In a clinical sense it would be impossible to gauge two devices under the exact same set of circumstances.

Finally, the use of computational techniques enables MR conditional safety to be evaluated for device prototypes. This is in contrast to clinical or experimental techniques, where the wait for a particular AIMD to be manufactured represents a delay in the timeline to evaluate the safety of a particular product. Thus, the use of CHMs in the assessment of MR conditional safety of AIMDs speeds up the development cycle and allows for new products to be developed specifically to meet MR conditional safety guidelines. Overall, this improves and accelerates the patient's access to both the benefits of MRI and to new therapies (provided by new AIMDs). 


\subsection{Evaluation of RF-Induced Malfunction Using Computational Human Models}

This work focuses on the RF-induced energy incident on the RF antenna port, which may interfere with device operation. Manufacturers must perform a safety assessment of this hazard to determine the ability of the device to perform within its acceptable tolerances during and after the MRI. This assessment is performed via bench top testing, where the test conditions are conservatively computed through the use of CHMs. The voltage is induced at the antenna port simultaneously with any voltage which is developed at the lead port.

At the lead ports, AIMD manufacturers follow the well-known transfer function method $[8,9]$ to conservatively estimate these induced voltages according to the equation

$$
V_{\mathrm{DUT}}=A \int_{0}^{L} S(\tau) \cdot E_{\mathrm{tan}}(\tau) d \tau
$$

A typical orientation of the implant for a dual-chamber pacemaker, with implantable pulse generator (IPG) location as well as atrial and ventricular pathways is shown in Fig. 14.2. A library of CHMs spanning the population in terms of height and BMI in different body positions, MR coils, and landmark positions is used to study the distribution of expected electromagnetic fields along the lead pathway. These fields (specifically, the component of the electric field tangent to the lead pathway) are then used to predict the response at the entry points to the cardiac implantable electronic device (CIED) by using a transfer function as a lead model.

Lead models are developed experimentally [10], in one or more tissue simulating media (TSM). The homogenous TSM should be chosen to accurately compute the RF-induced voltage once the transfer function is applied in the human body (via CHM).

Fig. 14.2 Example orientation of dualchamber pacemaker in the human body

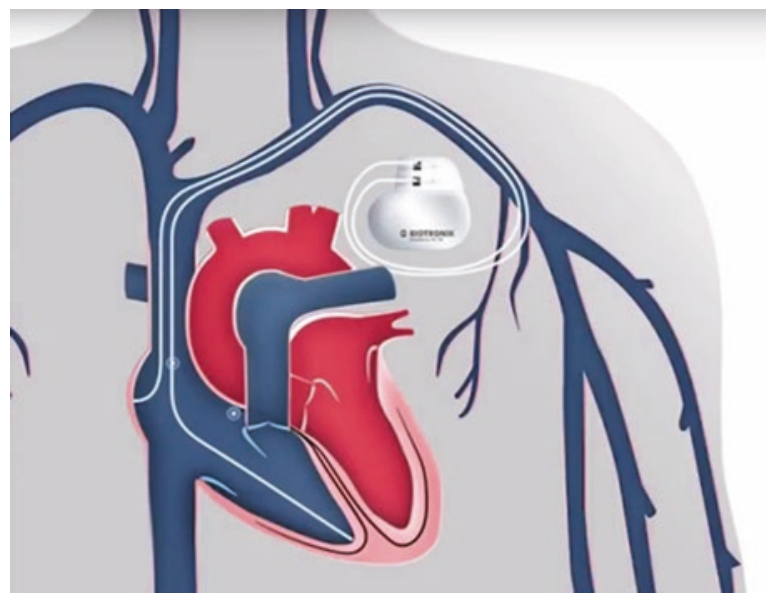


The geometric accuracy of the CHM along the lead trajectory, including the continuity of organs through which the leads are placed, is paramount to the accuracy of the model. Variations in critical parameters such as surrounding anatomy and tissue properties must be included in the set of simulations used to generate the worst-case predicted RF-induced energy.

After deriving a probability distribution of the RF-induced energy at the AIMD (which can be expressed in terms of voltage, current, or power), the device is injected via bench-top test. The value from the distribution is chosen using a riskbased analysis and scaled by appropriate uncertainties to provide a conservative analysis of the device performance during MRI. The device is monitored to ensure it operates within its acceptable range during the injection and a post-test further examines the AIMD to ensure no damage has occurred to any of the device's subsystems.

The developing standard for leaded CIEDs establishes a test method for performing the RF-induced malfunction assessment of the antenna port [3]. Though this standard has been developed for these devices, it is extensible to devices of similar construction, such as spinal cord stimulators and deep brain stimulators. The standard method [3] consists of modeling the AIMD in two phantoms, high permittivity medium (HPM) and low permittivity medium (LPM), and exposing it to a uniform E-field oriented along each of the three axes, for a total of six models to derive a coupling coefficient (i.e., a scalar which gives the RF-induced voltage due to a $1 \mathrm{~V} / \mathrm{m}$ incident field in the given direction). Separately, the expected peak E-field at the device location is derived by simulating a set of CHMs within a series of RF birdcage coils. Finally, the test condition for bench top RF injection testing is derived by multiplying this coupling coefficient with the predicted in vivo E-field according to the equation

$$
V_{\text {ant }}=E_{\text {scanner }} \sqrt{A_{x}^{2}+A_{y}^{2}+A_{z}^{2}}+B_{\text {scanner }} \sqrt{C_{x}^{2}+C_{y}^{2}+C_{z}^{2}} .
$$

This method involves separately simulating the CHM without the device present, simulating the device in a phantom and combining the results. A flowchart illustrating the process is shown in Fig. 14.3.

\subsection{Approach}

This work explores the accuracy of the method compared to a more rigorous model containing both the implanted device geometry and the birdcage coil. That method is more computationally intensive than performing the simulations separately, and is thus less desirable, as all of a large number of CHM-birdcage coil models would need to be resolved for every device. The method is explored for a pacemaker-type device but could be applied to other AIMDs of similar construction. 


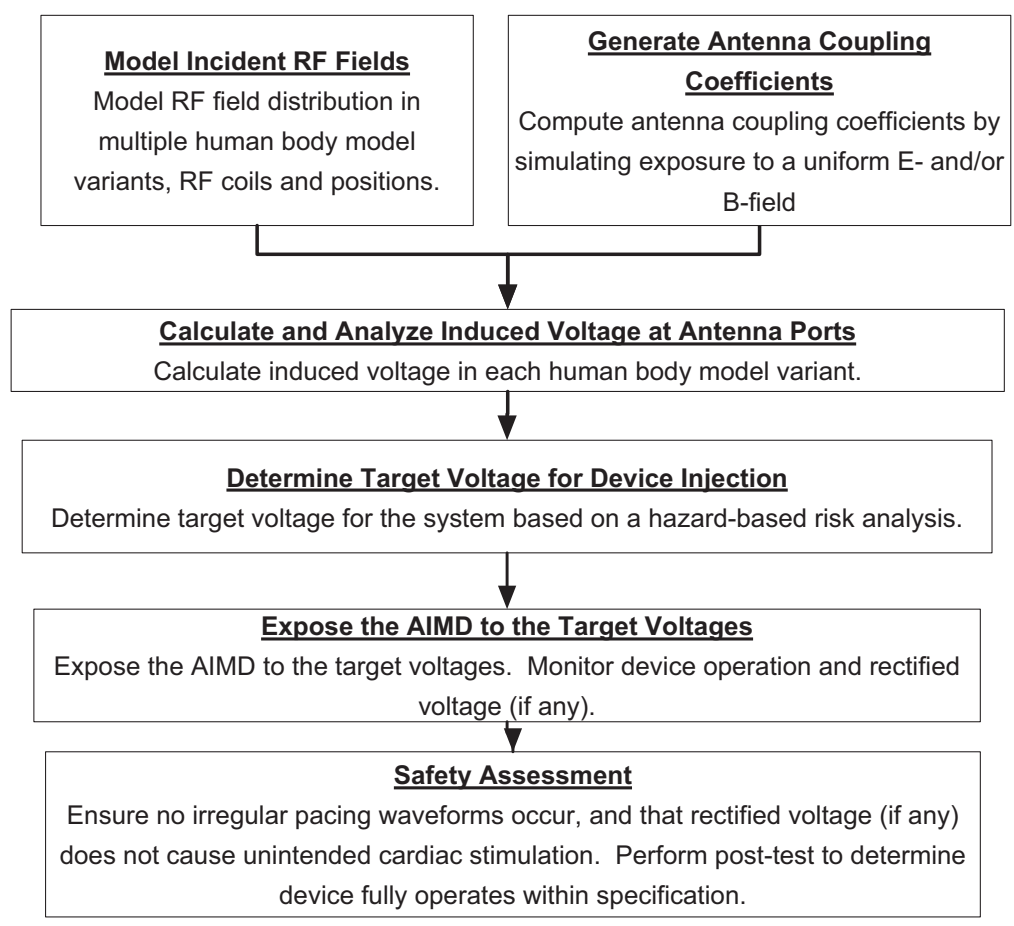

Fig. 14.3 Flow chart illustrating workflow for the assessment of protection from harm to the patient caused by RF-induced malfunction and rectification

\subsubsection{Computational Human Model}

A full device safety assessment would likely include a variety of human body models in a number of scan positions (e.g., supine, prone, etc.) and landmark locations (e.g., eyes, hips, or other anatomical region at isocenter) with respect to the MR coil geometry. The methods in this work are demonstrated by using one CHM in one scan position in one $3 \mathrm{~T}$ coil and are extensible to a full range of conditions for a device safety assessment.

The CHM used in this study is the Visible Human Project (VHP)-Female v.3.0 [11]. The subject is $162 \mathrm{~cm}$ in height, with BMI of about 33.5. For electromagnetic simulation, this model has been shown to be computationally efficient and platform independent. In order to prevent CAD import errors, techniques such as using fully enclosed objects to avoid Boolean subtractions were used in the development of the model.

The CHM is shown in Fig. 14.4. The IPG is placed in the left pectoral region. Pacemaker implantation is usually in the area between fat and pectoral muscle on either the left or right side. The incident electric field would necessarily be very dif- 
Fig. 14.4 The VHP-

Female v. 3.0 model used in this study

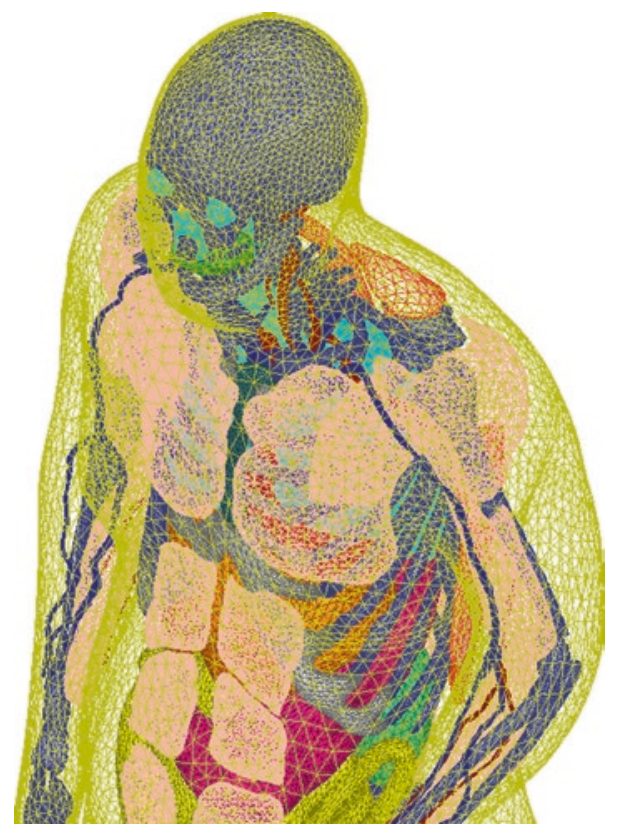

ferent within these two tissues, due to the high contrast in permittivity. The device placement intersects these materials and a Boolean subtraction is performed with the device as the tool object. Objects in this example which require these subtractions include the pectoralis major and rib cartilage. This is due to the inability to actually deviate the anatomy around the implant and potentially represents a source of error between these computational techniques and the in vivo condition.

\subsubsection{Device Model}

The antenna used in this study is a loop antenna (classified as a B-field coupled antenna in [3]) in an epoxy header over a metallic enclosure. As this work does not consider optimization of the antenna, the model was constructed after the antenna used in [12]. The dimensions of the antenna are chosen for operation in the $2.4 \mathrm{GHz}$ industrial, scientific, and medical (ISM) radio band, which is a higher frequency than the RF field of the MR system. The device is shown in Fig. 14.5. The can (device base) is a hollow (air-filled) PEC box. The header is modeled as epoxy and the feedthrough is ceramic. The antenna terminals are left open-circuited and the voltage drop is calculated between the two terminals inside the can. 
Fig. 14.5 The device model used for this work, which is meant to represent a pacemaker geometry and includes a loop antenna

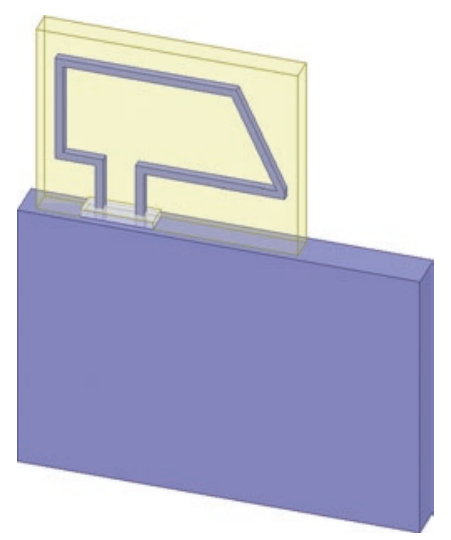

\subsubsection{MRI Birdcage Coil Model}

Two sets of simulations were performed using ANSYS Electronics Desktop. The 3T birdcage coil model was implemented using electromagnetic and circuit cosimulation [13], while the phantom models require only electromagnetic simulation. After solving the $3 \mathrm{~T}$ birdcage coil model, the incident field was linked to a model including the CHM within the bore. Two simulations were then performed one with and one without the device present. When the device was not present, some $2 \mathrm{D}$ and $3 \mathrm{D}$ regions were included to ease the computation of the incident field at the implant location. These included the rectangular solids shown in Fig. 14.5, for the can and header volume, as well as $2 \mathrm{D}$ sheets centered in these respective volumes.

\subsubsection{Uniform Field Excitation}

As the antenna in this study is a B-field coupled antenna, the test procedure outlined in [3] requires the exposure of the device to a uniform incident B-field. In order to expose the device to this B-field, a set of dual plane wave simulations were performed using ANSYS HFSS. By configuring each plane wave to have the same magnitude, a standing wave is generated in the modeled space which leads to cancellation of the E-field and uniform B-field in a particular direction. In this way, the RF-induced voltage at the antenna terminals may be extracted and normalized to a $1 \mu \mathrm{T}$ incident field.

The phantom is a cube, $100 \mathrm{~cm}$ wide on each edge. The cube is assigned to be alternately the $\operatorname{LPM}\left(\varepsilon_{\mathrm{r}}=11.5, \sigma=0.045\right)$ and the $\operatorname{HPM}\left(\varepsilon_{\mathrm{r}}=78, \sigma=0.47\right)$ material properties as described in [3]. The model is extended by an enclosing vacuum object which is $300 \mathrm{~cm}$ on each side. The radiation boundary is assigned to the vacuum object. The phantom, with an example of one B-field orientation and the device model placement, is shown in Fig. 14.6. 


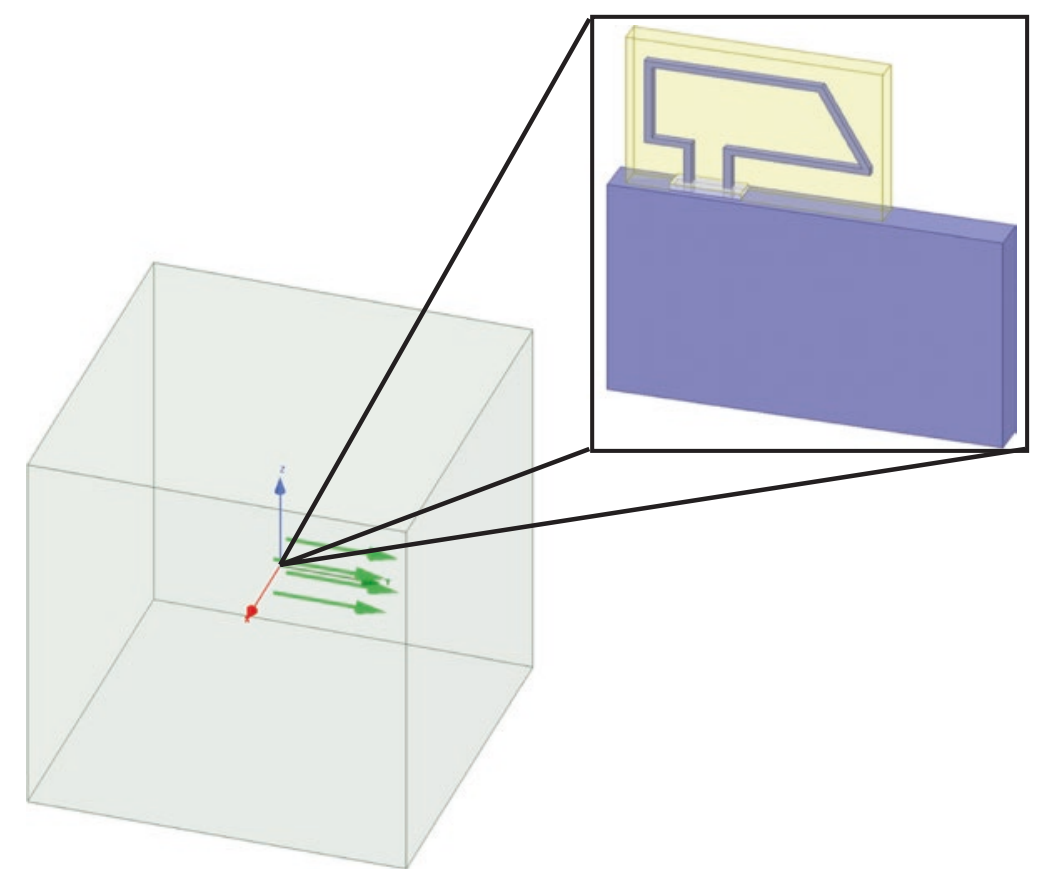

Fig. 14.6 The phantom model used for this study. The magnetic field is shown oriented in the $y$-direction at the device location. The device is not shown to scale with the phantom dimensions

\subsection{Results}

The results of the method in [3] and the more complete model are compared with each other. The field distribution of the CHM without the device present is then combined with these coefficients to give the predicted induced voltage at the antenna terminal. In order to quantify the field distribution at the CHM location, a number of methods are used. These are to take either the peak or the average fields values in a plane coinciding with the central axis of the device, the entire device volume, and the header volume. The non-model $2 \mathrm{D}$ and $3 \mathrm{D}$ objects described in the previous section are used to calculate volume and area averages for the relevant objects.

These six field distribution quantities are then combined with the results from either of the two phantoms, where the six coupling coefficients using Eq. 14.2 then give a voltage to be compared with the more rigorous and computationally expensive method of modeling the device in the CHM. These 12 results are compared to the calculated value in Fig. 14.7. In the figure, the term "device" means "can and header."

The results show that a number of these methods give a very conservative result. In fact, care should be taken to not overestimate the induced voltage, as this would lead to overly conservative test conditions. 


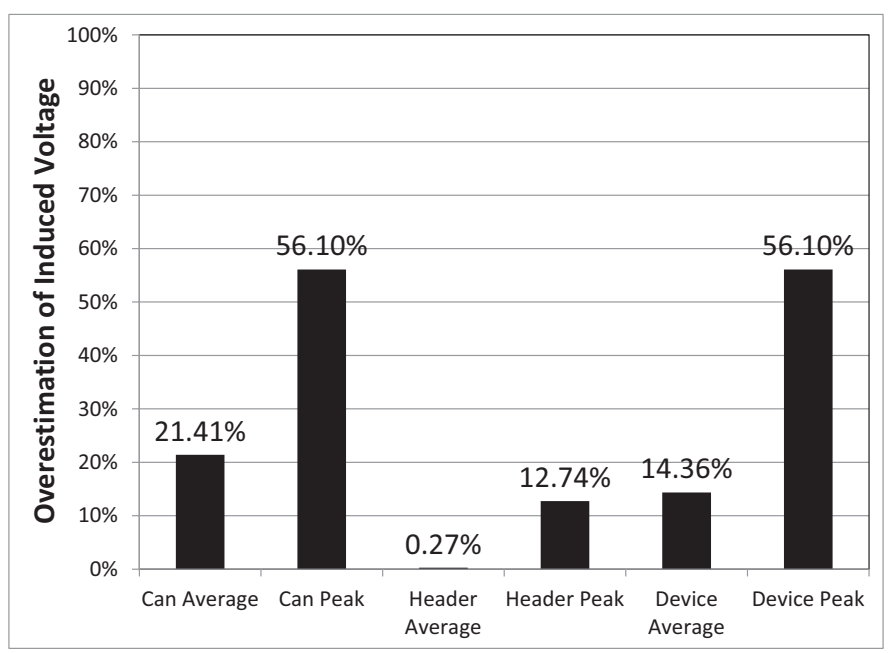

Fig. 14.7 The estimated RF-induced voltage from the HPM phantom model compared to the actual voltage from the CHP simulation. Different methods of quantifying the incident field distribution at the implant location result in varying degrees of overestimation of the induced voltage. "Device" refers to the combination of the can and header

A risk analysis for a device safety assessment following a process outlined in Fig. 14.1 would require many more simulations and a larger database of incident field distributions from the birdcage coil.

All investigated methods were conservative in estimating the induced voltage calculated via the more computationally expensive method for the case investigated. The method outlined in [3] and presented by Eq. (14.1) assumes worst-case alignment of the incident field vector and the antenna coupling coefficient, and thus is assumed to always be conservative. Additionally, the method requires taking the worst-case result from two phantoms, which further ensures the results to be conservative to the in vivo case.

\subsection{Future Work}

In the future, this work will be extended to $1.5 \mathrm{~T}$ MR systems. While this work considered only a loop (B-field coupled) antenna, E-field coupled antennas, as well as more complex structures (e.g., helices), may be investigated. Additionally, the results presented here are for a single CHM, and a wider anatomical variety spanning the range of the patient population should be considered. Further, the impact of variability of the real situation such as the patient's position within the bore, variations in coil design, etc., can be considered as well. Finally, shimming techniques [7], which are used in 3T MR systems to improve field homogeneity, can be investigated. For this work, only one excitation vector for the coil ports was used. 
There are many challenges related to the combination of modeling an MR coil, CHM, and device geometry in a single simulation. While some studies of these techniques exist $[14,15]$, the authors identified challenges related to computational resources for these efforts. These challenges arise from the fine details of the device model being solved in the large simulation volume of the CHP, MR coil, and surrounding space.

In the future, this work should be extended to realistic AIMD IPG geometries, as well as including leads. Lead geometries are sometimes coiled tightly (this is especially true for CIEDs) and include multiple conductors within a small volume. In addition, the impact of multiple leads on the induced voltages should be studied. Device operation can be incorporated through linking the electromagnetic simulation to a circuit model. The bulk of the literature in this area focuses on the analysis of RF-induced heating near leads for homogenous computational phantoms using computational techniques such as the method of moments [16] and the finite element method [17, 18]. Many of these techniques are extensible to calculating RF-induced energy at the proximal end of the lead as well. To date, in-depth device modeling has not been extensively performed in CHMs because of the challenges related to lead geometries. Future work could enable the evaluation of heat-reducing lead designs in realistic anatomies, instead of inhomogeneous phantoms, as is done in the literature today [19]. The further development of CHMs to provide optimal conditions for device modeling is an enabling technology.

Finally, the impact of any observed rectified voltage can be considered by incorporating a physiological model at the electrode interface to determine the probability of unintended stimulation, if any. Increased model development may further improve this area, as simulation could be used to speed the development of the physiological model. In particular, a Medical Device Development Tool could include co-simulation of electromagnetic and physiological phenomena [6]. Additionally, evaluation of, for example, unintended cardiac stimulation due to RF-induced rectified voltage, could be investigated via co-simulation rather than through developing probability models based on extensive lead testing.

\section{References}

1. Kalin, R., \& Stanton, M. S. (2005). Current clinical issues for MRI scanning of pacemaker and defibrillator patients. Pacing and Clinical Electrophysiology, 28(4), 326-328.

2. ISO/TS 10974:2018, "Assessment of the safety of magnetic resonance imaging for patients with an active implantable medical device".

3. AAMI PC76 (Draft), "Requirements and Test Protocols for Safety of Patients with Pacemakers and ICDs Exposed to MRI", to be published.

4. Brown, J. E., et al. (2016). MR conditional safety assessment of implanted medical devices: Advantages of computational human phantoms. Proceedings of the 38th Annual International Conference IEEE EMBC, Orlando, pp. 6465-6468.

5. Christ, A., et al. (2010). The Virtual Family—development of surface-based anatomical models of two adults and two children for dosimetric simulations. Physics in Medicine and Biology, $55, \mathrm{~N} 23-\mathrm{N} 38$. 
6. US FDA. (2013). Medical Device Development Tools - Draft Guidance for Industry, Tool Developers, and Food and Drug Administration Staff.

7. Ibrahim, T. S., et al. (2000). Application of finite difference time domain method for the design of birdcage RF head coils using multi-port excitations. Magnetic Resonance Imaging, 10, 733-742.

8. Park, S.-M., et al. (2007). Calculation of MRI-induced heating of an implanted medical lead wire with an electric field transfer function. Journal of Magnetic Resonance Imaging, 26, $1278-1285$.

9. Feng, S., et al. (2015). A technique to evaluate MRI-induced electric fields at the ends of practical implanted lead. IEEE Transactions on Microwave Theory and Techniques, 63(1), 305-313.

10. Zastrow, E., Capstick, M., \& Kuster, N. (2016). Experimental system for RF-heating characterization of medical implants during MRI. Proceedings of the 24th Annual Meeting ISMRM, Singapore.

11. Noetscher, G. M., et al. (2016). Computational human model VHP-female derived from datasets of the National Library of Medicine. Proceedings of the 38th Annual International Conference IEEE EMBC, Orlando, pp. 3350-3353.

12. ETSI TR 102655 v. 1.1.1. (2008). Electromagnetic compatibility and Radio spectrum Matters (ERM); System reference document; Short Range Devices (SRD); Low Power Active Medical Implants (LP-AMI) operating in a $20 \mathrm{MHz}$ band within $2360 \mathrm{MHz}$ to $3400 \mathrm{MHz}$.

13. Bonmassar, G., Serano, P., \& Angelone, L. M. (2013). Specific absorption rate in a standard phantom containing a deep brain stimulation lead at 3 Tesla MRI. Proceedings of the 6th International IEEE/EMBS Conference NER, San Diego, CA, pp. 747-750.

14. Zastrow, E., Cabot, E., \& Kuster, N. (2014, August). Assessment of local RF-induced heating of AIMDs during MR exposure. XXXIth URSI GASS, Beijing, China.

15. Cabot, E., Zastrow, E., \& Kuster, N. (2014, August). Safety assessment of AIMDs under MRI exposure: Tier3 vs. Tier4 evaluation of local RF-induced heating. International Symposium EMC, Tokyo.

16. Brown, J. E. (2012). Radiofrequency heating near medical devices in magnetic resonance imaging. Ph.D. dissertation, Bobby B. Lyle School of Engineering, Southern Methodist University, Dallas.

17. Brown, J. E., \& Lee, C. S. (2013). Radiofrequency resonance heating near medical devices in magnetic resonance imaging. Microwave and Optical Technology Letters, 55(2), 299.

18. McCabe, S.O., \& Scott, J.B. (2014, November). Cause and amelioration of MRI-induced heating through medical implant lead wires. 21st Elect New Zealand Conference, Hamilton.

19. Nordbeck, P., et al. (2012). Reducing RF-related heating of cardiac pacemaker leads in MRI: Implementation and experimental verification of practical design changes. Magnetic Resonance in Medicine, 68, 1963-1972.

Open Access This chapter is licensed under the terms of the Creative Commons Attribution 4.0 International License (http://creativecommons.org/licenses/by/4.0/), which permits use, sharing, adaptation, distribution and reproduction in any medium or format, as long as you give appropriate credit to the original author(s) and the source, provide a link to the Creative Commons license and indicate if changes were made.

The images or other third party material in this chapter are included in the chapter's Creative Commons license, unless indicated otherwise in a credit line to the material. If material is not included in the chapter's Creative Commons license and your intended use is not permitted by statutory regulation or exceeds the permitted use, you will need to obtain permission directly from the copyright holder.

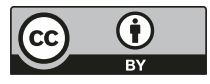

